\title{
Investigating the thermodynamics and susceptibilities of the $(2+1)$ Polyakov quark meson model
}

\section{Sandeep Chatterjee*}

Centre for High Energy Physics, Indian Institute of Science, Bangalore, India

E-mail: sandeepects.iisc.ernet.in

\section{Kirtimaan A. Mohan}

Centre for High Energy Physics, Indian Institute of Science, Bangalore, India

E-mail: kirtimaan@ets.iisc.ernet.in

\begin{abstract}
We consider the $(2+1)$ flavor Polyakov Quark Meson Model (PQM) and study the effect of including fermion vacuum fluctuations on the thermodynamics and phase diagram. The variation of the thermodynamic quantities across the phase transition region becomes smoother. This results in better agreement with the lattice QCD (LQCD) data. The critical end point is pushed into higher values of the chemical potential. We then go on to study the fluctuations of conserved charges in this model up to sixth order. Comparison is made with LQCD wherever available and overall good qualitative agreement is found, more so for the case of the normalized susceptibilities. Our study provides a solid basis for the use of PQM as an effective model to understand the topology of the QCD phase diagram.
\end{abstract}

Sixth International Conference on Quarks and Nuclear Physics,

April 16-20, 2012

Ecole Polytechnique, Palaiseau, Paris

\footnotetext{
*Speaker.
} 


\section{Introduction}

Lattice QCD (LQCD) techniques have given us important insights into various aspects of the phase transitions of a QCD medium. However, the notorious sign problem at non zero baryon density prevents us from eliciting precise information about QCD phase transitions. Although several methods have been developed [1] to bypass the sign problem at small baryon chemical potential, a satisfactory solution for all values still eludes us.

An alternative is to study various phenomenological models which behave very similar to QCD and can at least qualitatively if not quantitatively mimic QCD. These models serve to complement LQCD computations and also provide us with an intuitive and physical understanding about the behavior of the phases of strongly coupled matter in regions that are both accessible and inaccessible to LQCD with minimal effort.

Fluctuations and correlations of the conserved charges can provide signatures of the critical end point (CEP) [2] and illuminate the structure of the QCD phase diagram. The great advantage that these observables provide is that they can also be extracted experimentally through event by event analysis making them important observables in understanding the nature of strongly interacting matter [3,4].

There have been numerous studies of QCD correlators and thermodynamics, both in lattice as well as models [5-9]. We present some results of our study in the framework of the $2+1$ flavor PQM (Polyakov-Quark-Meson) [8] model and its subsequent improvement with the inclusion of the vacuum term (PQMVT) [10].

\section{Thermodynamics of the PQM Model}

The relevant thermodynamic potential at a temperature $T$ and chemical potentials $\mu_{B}, \mu_{Q}$ and $\mu_{S}$ in the mean field approximation can be written as $[10,11]$

$$
\Omega\left(T, \mu_{B}, \mu_{Q}, \mu_{S}\right)=\mathscr{U}_{\mathrm{M}}\left(\sigma_{u}, \sigma_{d}, \sigma_{s}\right)+\mathscr{U}_{\mathrm{Poly}-\mathrm{VM}}(\Phi, \bar{\Phi}, T)+\Omega_{\bar{q} q}\left(T, \mu_{B}, \mu_{Q}, \mu_{S}, \sigma_{u}, \sigma_{d}, \sigma_{s}, \Phi, \bar{\Phi}\right)
$$

where $\mathscr{U}_{\mathrm{M}}$ and $\mathscr{U}_{\text {Poly-VM }}$ are the contributions from the mesonic sector and the Polyakov loop respectively. The quark/antiquark contribution is given by

$$
\Omega_{\bar{q} q}\left(T, \mu_{B}, \mu_{Q}, \mu_{S}, \sigma_{u}, \sigma_{d}, \sigma_{s}, \Phi, \bar{\Phi}\right)=\Omega_{\bar{q} q}^{\mathrm{v}}\left(\sigma_{u}, \sigma_{d}, \sigma_{s}\right)+\Omega_{\bar{q} q}^{\mathrm{th}}\left(T, \mu_{B}, \mu_{Q}, \mu_{S}, \sigma_{u}, \sigma_{d}, \sigma_{s}, \Phi, \bar{\Phi}\right)
$$

where $\Omega_{\bar{q} q}^{\text {th }}$ is the contribution due to thermal fluctuations and $\Omega_{\bar{q} q}^{\mathrm{v}}$ is the vacuum term

$$
\begin{aligned}
\Omega_{\bar{q} q}^{\mathrm{v}}\left(\sigma_{u}, \sigma_{d}, \sigma_{s}\right) & =-2 N_{c} \sum_{f=u, d, s} \int \frac{d^{3} p}{(2 \pi)^{3}} E_{f} \\
& =-\frac{N_{c}}{8 \pi^{2}} \sum_{f=u, d, s} m_{f}^{4} \log \left[\frac{m_{f}}{\Lambda}\right]
\end{aligned}
$$

where $\Lambda$ is the regularisation scale. We study the influence of the vacuum term on the thermodynamics of the PQM model. For further details regarding the model we refer the reader to [10]. Fig. (1) shows the chiral condensates $\left\langle\sigma_{x}\right\rangle$ (corresponding to light quarks), $\left\langle\sigma_{y}\right\rangle$ (corresponding to 

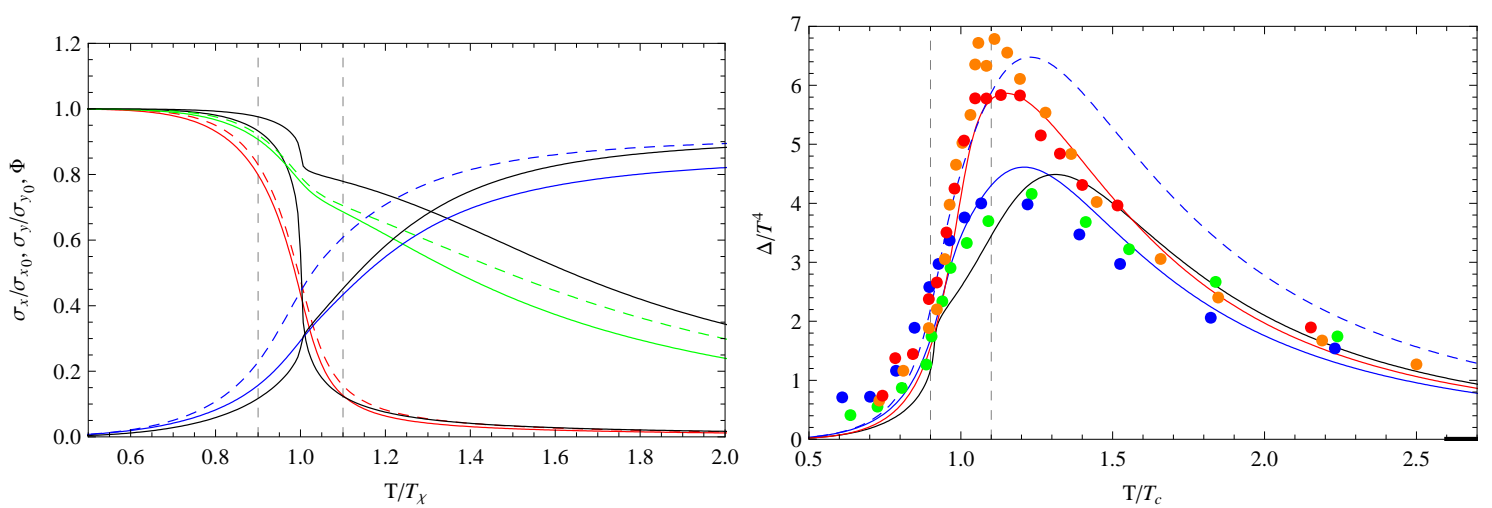

Figure 1: Left:Plot of normalized $\left\langle\sigma_{x}\right\rangle$ (red), $\left\langle\sigma_{y}\right\rangle$ (green) and $\langle\Phi\rangle$ (blue) vs $T / T_{\chi}$ at $\mu_{x}=\mu_{y}=0$ as obtained in PQMVT. The dashed curves refer to ModelHotQCD while the solid curves refer to ModelWB. The solid black curve in each case is obtained in PQM with the ModelWB parameter set. Right: $\Delta$ in PQMVT with ModelWB (blue solid) and ModelHotQCD (red solid) parameter sets. The corresponding black curves are the PQM predictions with ModelWB parameter set. For comparison, model predictions with the Polyakov potential without the VanderMonde term and ModelWB parameter set are also shown in dashed blue. Here $T_{c}=\frac{T_{\chi}+T_{d}}{2}$. The LQCD data from WB and HotQCD are shown, for details refer [10]

strange quarks) and the Polyakov loop field $\langle\Phi\rangle$ obtained with two different parameter sets which we refer to as ModelHotQCD and ModelWB at zero chemical potentials. These parameter sets differ only in the values of $T_{0}$ and $\kappa$ that are parameters of the Polyakov loop potential [10]. For ModelHotQCD $T_{0}=210 \mathrm{MeV}$ and $\kappa=0.1$ while for ModelWB $T_{0}=270 \mathrm{MeV}$ and $\kappa=0.2$. We see that using a higher value of $T_{0}$ and $\kappa$ in ModelWB results in a delayed confinementdeconfinement crossover as well as a suppressed value for the Polyakov condensate as compared to ModelHotQCD. In the absence of the vacuum term , the $\left\langle\sigma_{x}\right\rangle$ transition is much sharper and is accompanied by an unsmooth structure in $\left\langle\sigma_{y}\right\rangle$ and $\langle\Phi\rangle$.

The model predictions for the conformal symmetry breaking measure $\Delta$ has also been shown in the right panel of Fig. (1) and compared with LQCD. In this case we have plotted $\Delta$ against $T_{c}=\frac{T_{\chi}+T_{d}}{2}$, where $T_{\chi}$ is the chiral crossover temperature and $T_{d}$ is the deconfinement transition temperature, for better agreement between model and LQCD. As mentioned in [10] we have chosen the value of $\kappa$ such that the model predictions agree well with LQCD. In general $\kappa$ could be taken as a function of $T$ but in this work we have taken a constant value for $\kappa$.

The unsmooth structures in $\left\langle\sigma_{y}\right\rangle$ and $\langle\Phi\rangle$ that we found around $T_{\chi}$ in the case of PQM shows up in the case of $\Delta$ also. As observed in Fig. (1), addition of the vacuum term in PQMVT results in a smooth behavior of the condensates in the transition region which in turn smoothen all the thermodynamic quantities.

The divergence of the second order quark number susceptibility $\chi_{2}^{u}=\frac{\partial^{2}\left(p / T^{4}\right)}{\partial\left(\mu_{u} / T\right)^{2}}$, where $p$ is the pressure and $\mu_{u}$ is the chemical potential for the light quark, can be used to locate the CEP [3]. We obtain the phase diagram in the case of symmetric quark matter $\left(\mu_{x}=\mu_{y}=\mu\right)$. The smoothening of the thermodynamic quantities in the transition region as seen for $\mu=0$ persists even at non-zero chemical potentials. This results in pushing the CEP to a higher value of $\mu$ in case of PQMVT as compared to PQM. When we use larger values of the sigma meson mass, $m_{\sigma}=600$, the mesonic potential does not possess the necessary structure for symmetry breaking. As a result the phase 
diagram has no CEP and there is only crossover transition on the entire $(\mu-T)$ plane as in the case of 2 flavors [15].

\section{Fluctuations and correlations}

In order to find the generalized susceptibilities of the conserved charges, one has to take appropriate derivatives of $P$

$$
\chi_{i j k}^{B Q S}=\frac{\partial^{i+j+k}\left(P / T^{4}\right)}{\partial\left(\mu_{B} / T\right)^{i} \partial\left(\mu_{Q} / T\right)^{j} \partial\left(\mu_{S} / T\right)^{k}}
$$

At zero chemical potential, the baryon number, electric charge, and strangeness densities vanish while the higher order derivatives with $(i+j+k)$ even, are nonzero. We compute $\chi_{i j k}^{B Q S}$ up to $(i+j+k)=6$ order at zero chemical potential. The derivatives in (3.1) have been computed using the algorithmic differentiation techniques available in ADOL-C [16] which allow us to compute higher order derivatives efficiently and without additional truncation errors. At low temperatures the large values of condensates leads to large masses of the relevant degrees of freedom resulting in small values for various susceptibilities. However as the temperature increases the condensates begin to melt and we should expect that the susceptibilities acquire higher values as we approach $T_{c}$.

Well beyond $T_{c}$, the system is expected to behave like a Stefan-Boltzmann (SB) gas of 3 flavors of massless quarks. In contrast, at low temperatures the system is expected to behave like an ideal gas of hadrons as exhibited in the Hadron Resonance Gas Model (HRGM) [11]. LQCD data from WB [17] exhibit the above expectations quite clearly. Although the PQM model does not have baryons, there are 'three quark' states which mimic baryonic degrees of freedom in the low $T$ limit, one thus expects the model predictions to match with those of HRGM to some extent.

In Fig. (2) we present some generalized susceptibilities and correlations computed in PQMVT at zero chemical potentials and compare with LQCD data [17-21]. We see that the model predictions not only match lattice data very well but at low temperatures they also match HRGM predictions. The model predictions also saturate to the SB limit at high $T$.

All second order diagonal susceptibilities, as expected, are small at low $T$ but increase as they approach $T_{c}$ and then saturate to the SB limit at high $T$. At low $T$, ratios of the kind $\chi_{i}^{X} / \chi_{j}^{X}$ where $X \in B, Q, S$ approach unity both in PQM as well as in HRGM [10]. For fourth order susceptibilities, in the crossover region, all the ratios shown in Fig. (2) exhibit a peak, the most prominent being that of $\chi_{4}^{B} / \chi_{2}^{B}$. On the other hand, sixth order ones oscillate between positive and negative values passing through zero once in the transition region. This feature is expected since at zero chemical potential in the chiral limit, the singular behavior of the quadratic and higher order baryon number fluctuations are supposed to be controlled by the $O(4)$ symmetry group with the scaling behavior $\chi_{2 n}^{B} \sim\left|\frac{T-T_{c}}{T_{c}}\right|^{2-n-\alpha}$ where $\alpha \simeq-0.25$ [22]. Therefore as we go to higher order susceptibilities the singularities that appear about $T_{c}$ become stronger leading to stronger fluctuations in higher order susceptibilities.

We also show off-diagonal susceptibilities normalized by diagonal ones in the third row of Fig. (2). In this case the high $T$ regime $\chi_{11}^{B Q} / \chi_{2}^{B}$ goes to zero as $\chi_{11}^{B Q}$ itself goes to zero. Note that the $\chi_{11}^{B Q}$ gets contributions from protons whereas $\chi_{2}^{B}$ gets contributions from both protons and 

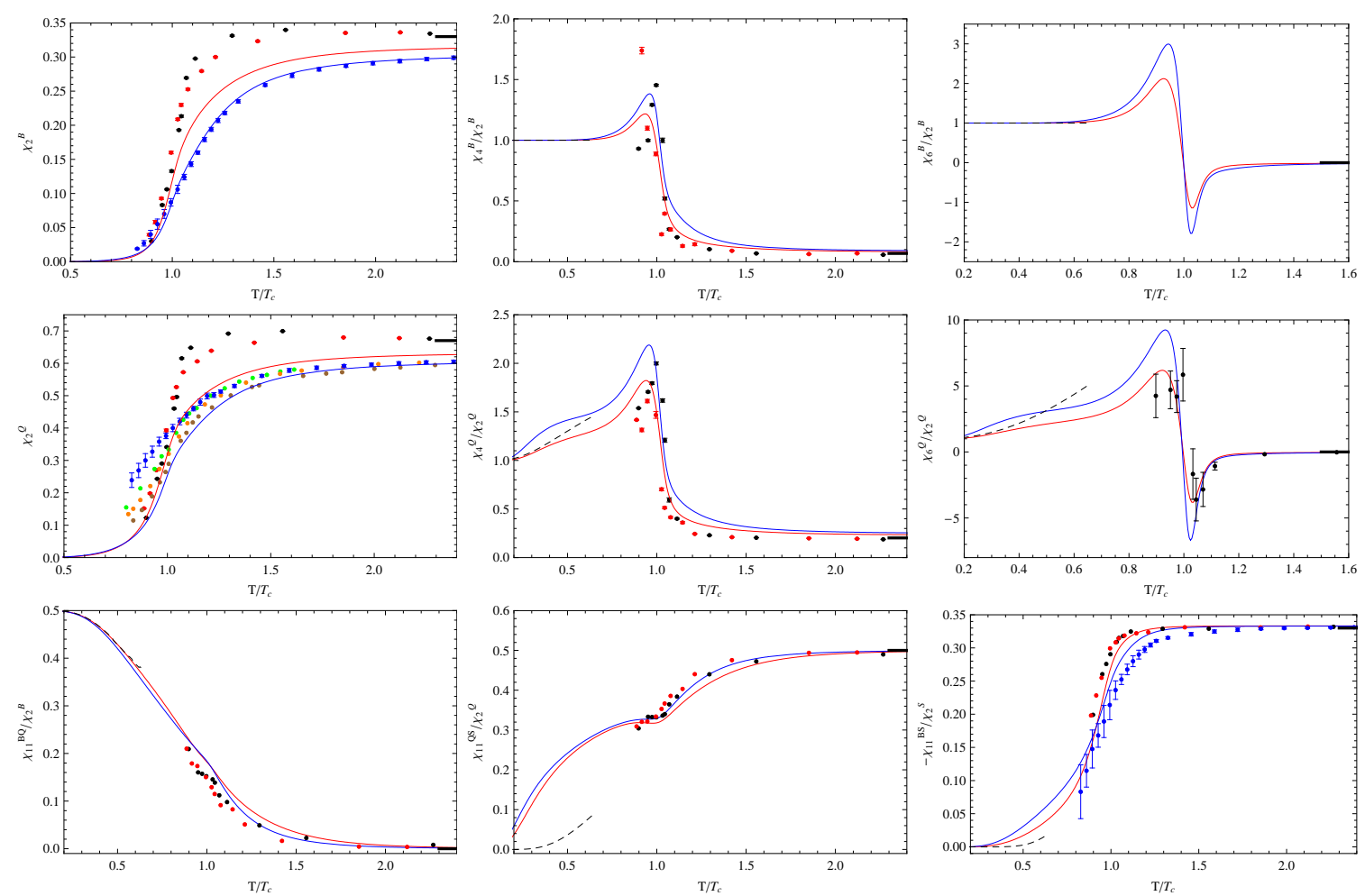

Figure 2: Row 1: From left to right, $\chi_{2}^{B}, \chi_{4}^{B} / \chi_{2}^{B}$ and $\chi_{6}^{B} / \chi_{2}^{B}$. Row 2: From left to right, $\chi_{2}^{Q}, \chi_{4}^{Q} / \chi_{2}^{Q}$ and $\chi_{6}^{Q} / \chi_{2}^{Q}$. Row 3: From left to right, $\chi_{11}^{B Q} / \chi_{2}^{B}, \chi_{11}^{Q S} / \chi_{2}^{Q}$ and $-\chi_{11}^{B S} / \chi_{2}^{S}$. In all plots PQMVT-ModelHotQCD (in red) and PQMVT-ModelWB (in blue) predictions are shown. For details about lattice data refer [11].

neutrons at low temperatures we therefore see $\chi_{11}^{B Q} / \chi_{2}^{B}$ approaching $1 / 2$ as the temperature falls. $-\chi_{11}^{B S} / \chi_{2}^{S}$ on the other hand shows a steep rise. In the low $T$ limit, we expect this ratio to go to zero: since the three quark states from the strange sector (which dominantly contribute to $\chi_{11}^{B S}$ in PQM) are much heavier compared to kaons which dominantly contribute to $\chi_{2}^{S}$. Since kaons which contribute dominantly to $\chi_{11}^{Q S}$, are much heavier than pions that have a leading contribution to $\chi_{2}^{Q}, \chi_{11}^{Q S} / \chi_{2}^{Q}$ goes to zero as the temperature falls. $\chi_{11}^{Q S} / \chi_{2}^{Q}$ also exhibits an interesting feature in the transition regime, a plateau structure near $T_{c}$. Similar observation has been found even on the lattice [21] as well as in the case of PNJL [7] model. In [7] this has been attributed to the shift in the role of the dominant degrees of freedom from the hadrons to quark quasi-particles just above $T_{c}$.

Rapid variations in quantities such as $\chi_{11}^{B S} / \chi_{2}^{S}$ and $\chi_{11}^{Q S} / \chi_{2}^{Q}$ and in higher order susceptibilities make them well suited to probe the QCD phase transition. In fact, it was already pointed out in [4] that the ratio $\chi_{11}^{B S} / \chi_{2}^{S}$ has different values in the hadron and QGP phases.

\section{Conclusion}

It is encouraging to note that the PQMVT model agrees reasonably well with LQCD data. The model successfully reproduces many of the features that are seen in LQCD data in the transition regime both for thermodynamic quantities as well as susceptibilities. However, for $\chi_{2}^{Q}$ in the low 
$T$ limit, the model prediction is suppressed in comparison to LQCD. A possible reason for this is the exclusion of pionic fluctuations in mean field calculation. We also found that the ratios susceptibilities show very good agreement with LQCD data. Better agreement might be sought by going beyond the mean field as fluctuations may play an important role here [23]. It is also encouraging that the model successfully interpolates between a hadronic description (HRGM) and a free quark (SB) description of strong interactions. These factors provide a strong basis for the use of PQMVT as an effective model in the study of the QCD phase diagram.

\section{Acknowledgement}

S.C. would like to acknowledge discussions and collaborations with Sourendu Gupta for introduction to the subject itself. He would also like to thank B. -J. Schaefer for fruitful discussions on the subject. We would like to thank Rohini Godbole for guidance. K.A.M. acknowledges the financial support provided by CSIR, India.

\section{References}

[1] S. Gupta, PoS LATTICE 2010 (2010) 007 [arXiv:1101.0109 [hep-lat]].

[2] M. A. Stephanov et al., Phys. Rev. Lett. 81 (1998) 4816 [hep-ph/9806219].

[3] M. A. Stephanov et al., Phys. Rev. D 60 (1999) 114028 [hep-ph/9903292].

[4] V. Koch et al., Phys. Rev. Lett. 95 (2005) 182301 [nucl-th/0505052].

[5] K. Fukushima, Phys. Rev. D 77 (2008) 114028 [arXiv:0803.3318 [hep-ph]].

[6] W. -j. Fu et al., Phys. Rev. D 81 (2010) 014028 [arXiv:0910.5783 [hep-ph]].

[7] A. Bhattacharyya et al., Phys. Rev. D 83 (2011) 014011 [arXiv:1010.2394 [hep-ph]].

[8] B. -J. Schaefer et al., Phys. Rev. D 81 (2010) 074013 [arXiv:0910.5628 [hep-ph]].

[9] B. -J. Schaefer and M. Wagner, Phys. Rev. D 85 (2012) 034027 [arXiv:1111.6871 [hep-ph]].

[10] S. Chatterjee and K. A. Mohan, Phys. Rev. D 85 (2012) 074018 [arXiv:1108.2941 [hep-ph]].

[11] S. Chatterjee and K. A. Mohan, [arXiv:1201.3352 [hep-ph]].

[12] S. Borsanyi et al., JHEP 1011, 077 (2010) [arXiv:1007.2580 [hep-lat]].

[13] S. Borsanyi et al., arXiv:1109.5032 [hep-lat].

[14] A. Bazavov et al., Phys. Rev. D 80 (2009) 014504 [arXiv:0903.4379 [hep-lat]].

[15] U. S. Gupta and V. K. Tiwari, Phys. Rev. D 85 (2012) 014010 [arXiv:1107.1312 [hep-ph]].

[16] M. Wagner et al., Comput. Phys. Commun. 181 (2010) 756 [arXiv:0912.2208 [hep-ph]].

[17] S. Borsanyi et al., arXiv:1112.4416 [hep-lat].

[18] S. A. Gottlieb et al., Phys. Rev. Lett. 59 (1987) 2247.

[19] C. Bernard et al. [MILC Collaboration], Phys. Rev. D 71 (2005) 034504 [hep-lat/0405029].

[20] C. Bernard et al., Phys. Rev. D 77 (2008) 014503 [arXiv:0710.1330 [hep-lat]].

[21] M. Cheng et al., Phys. Rev. D 79 (2009) 074505 [arXiv:0811.1006 [hep-lat]].

[22] A. Pelissetto and E. Vicari, Phys. Rep. 368, 549 (2002)

[23] T. K. Herbst et al., Phys. Lett. B 696 (2011) 58 [arXiv:1008.0081 [hep-ph]]. 